REVIEW

\title{
Management of acute renal failure
}

\author{
A C Fry, K Farrington
}

Postgrad Med J 2006;82:106-1 16. doi: 10.1136/pgmj.2005.038588

Acute renal failure is a common condition, frequently encountered in both community practice and hospital inpatients. While it remains a heterologous condition, following basic principles makes investigation straightforward, and initial management follows a standard pathway in most patients. This article shows this, advises on therapeutic strategies, including those in special situations, and should help the clinician in deciding when to refer to a nephrologist, and when to consider renal replacement therapy.

See end of article for authors' affiliations

Correspondence to:

Dr A C Fry, Department of Renal Medicine, Lister Hospital, Coreys Mill Lane, Stevenage, Hertfordshire SG1 4AB, UK; andyfry@ doctors.org.uk

Submitted 17 June 2005 Accepted 3 August 2005
A cute renal failure (ARF) is a potentially reversible reduction in the capacity of the kidney to excrete nitrogenous wastes and maintain fluid and electrolyte homoeostasis, which usually occurs over hours to days. The initial clinical approach is identical in all patients-a thorough history and examination with simultaneous treatment of any life threatening features (for example, severe hyperkalaemia). Subsequent management should focus on determining the cause, which may demand specific treatment, maintaining the patient's volume status, and avoiding further nephrotoxic insults.

ARF is common, complicating 5\% of all medical and surgical admissions in a large American study. ${ }^{1}$ A British community based study, using a laboratory database to identify patients with an acute rise in serum creatinine (and subsequent decrease or patient death) found that the overall annual incidence of acute renal failure was 172 cases per million adult population. There was a significant increase with age: 17 cases annually per million population aged 16-50 years rising to 949 cases annually per million population in those aged $80-89$ years. $^{2}$ There is no standard definition of ARF, but common criteria are either biochemical (a rise in serum creatinine of $50 \mu \mathrm{mol} / \mathrm{l}$, or of $50 \%$ from baseline, or beyond a set level, for example, $>500 \mu \mathrm{mol} / \mathrm{l}$ ), or clinical (oliguria with urine output $<400 \mathrm{ml} /$ day), a combination of both, or the need for renal replacement therapy. ${ }^{34} \mathrm{~A}$ recent development is the RIFLE classification, which seeks to standardise definitions of ARF based on a combination of biochemical and urine output criteria. ${ }^{5}$

\section{CAUSES OF ACUTE RENAL FAILURE}

Classically, the causes of ARF have been subdivided into three groups: pre-renal, intrinsic, and post-renal. While there is considerable overlap between these, especially the first two, it remains a useful clinical guide (fig 1).

\section{Pre-renal}

Reduced renal perfusion, attributable to hypovolaemia, hypotension, or drugs, which is reversible on correction of the underlying cause.

\section{Intrinsic}

Commonly results from tubular cell injury or death attributable to prolonged, or inadequately corrected, pre-renal failure-ischaemic acute tubular necrosis (ATN). Drugs may also cause direct tubular damage-nephrotoxic ATN. Acute interstitial nephritis (AIN) may result from allergic drug reactions, infections, or occasionally systemic disease (for example, sarcoidosis). Fulminant glomerular inflammation-rapidly progressive glomerulonephritis (for example, because of vasculitis)-is the least common, but demands the most urgent investigation.

\section{Post-renal}

Obstruction of urine outflow of both kidneys, or a single functioning kidney, may arise anywhere from renal pelvis to urethra. Relief of obstruction usually leads to recovery of function.

Pre-renal failure is the most frequent cause, at least in hospitalised patients, although obstruction secondary to prostatic disease is as common in some community studies. ${ }^{2}$ Intrinsic disease is most probably attributable to ischaemic ATN (50\% of cases of intrinsic ARF), with nephrotoxic ATN, interstitial nephritis, and glomerulonephritis accounting for $35 \%, 10 \%$, and $5 \%$ of cases respectively. ${ }^{3}$ The condition is often multifactorial, for example, the septic, hypotensive patient given aminoglycosides and intravenous contrast. Elderly patients, diabetic patients, and those with pre-existing renal disease are all at higher risk.

\section{PATHOGENESIS}

Renal blood flow is 25\% of cardiac output but some areas are particularly sensitive to ischaemic damage. Most of the blood flow supplies the cortex, which contains the glomeruli and convoluted tubules, areas that require good perfusion to achieve filtration and reabsorption, the latter with high energy demands. The outer medulla is comparatively starved of oxygen, its blood supply first traversing the glomerular

Abbreviations: ARF, acute renal failure; ATN, acute tubular necrosis; AIN, acute interstitial nephritis; GFR, glomerular filtration rate; NSAID, non-steroidal antiinflammatory drug; ACE-I, angiotensin converting enzyme inhibitors; $A R B$, angiotensin receptor blockers; RRT, renal replacement therapy; CRRT, continuous renal replacement therapy; RPGN, rapidly progressive glomerulonephritis 


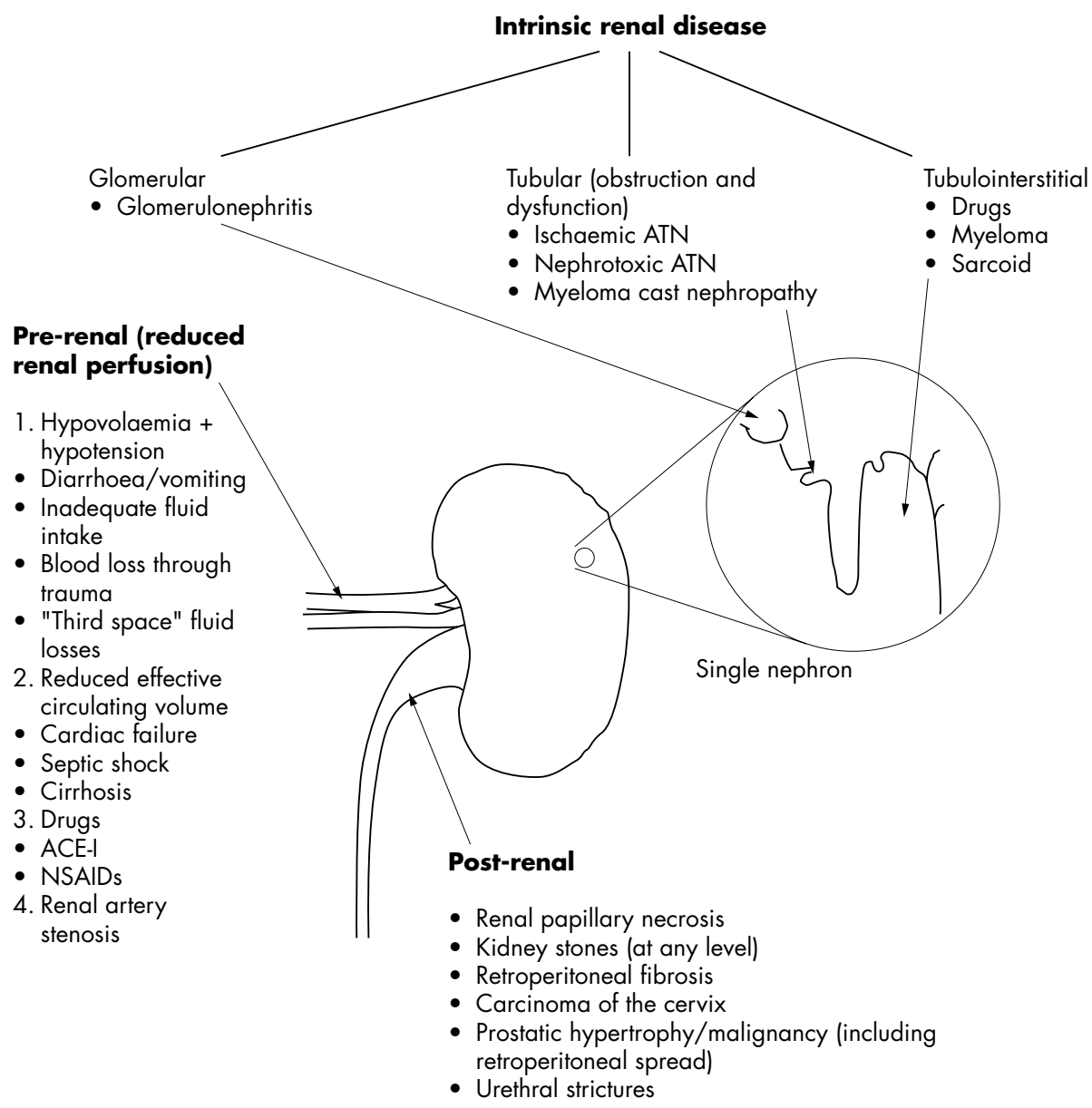

Figure 1 Aetiology of acute renal failure.

capillary bed, and losing hydrostatic pressure (in essence, a portal circulation), and then on entering the medulla, losing oxygen by countercurrent exchange with the venous vasa recta. These features are essential to maintain the osmotic gradients within the medulla and thus generate a concentrated urine, but render the outer medulla very susceptible to variations in blood flow. ${ }^{6}$ This area contains the thick ascending limb of the loop of Henle and S3 segment of the proximal tubule, both with high oxygen requirements. Impaired tubular sodium reabsorption attributable to reduced perfusion causes constriction of the afferent arteriole and a further reduction in glomerular filtration rate (GFR). This compensatory mechanism (tubuloglomerular feedback), designed to protect the downstream nephron, may cause injury if prolonged, or if normal regulation of the arterial tone is blocked (for example, by non-steroidal anti-inflammatory drugs (NSAIDs), angiotensin converting enzyme inhibitors (ACE-I) or angiotensin receptor blockers (ARBs)). Reduced blood flow in the peritubular capillaries produces ischaemic damage in vascular endothelial cells, resulting in cell swelling and the expression of cell adhesion molecules-reducing flow further and leading to leucocyte activation. Adherent leucocytes further impede blood flow and produce cytokines and reactive oxygen species that damage endothelial and tubular epithelial cells. Tubular cells swell, lose their brush border, and develop cytoskeletal abnormalities with abnormal localisation of cell membrane components (for example, $\mathrm{Na}+\mathrm{K}+-$ ATPase), changes in cellular polarity, and loss of cell-cell and cell-basement membrane attachment. These swollen, detached cells obstruct the tubular lumen, and backleak of filtrate occurs through the damaged basement membrane (fig 2). In the classic histological appearance of ATN, tubules are surrounded by flattened, denuded epithelium, and the lumen filled by cell debris, with congested peritubular capillaries and an extensive inflammatory cell infiltrate. $^{378}$ Cell death occurs predominantly by necrosis, although apoptosis also contributes-especially in the thick ascending limb and late in the process. ${ }^{7}$

A remarkable feature of the kidney is its ability to regain normal structure and function after such injury. Once renal perfusion and oxygen supply are normalised, viable cells still adherent to the tubular basement membrane can spread to cover denuded areas, and then differentiate to reproduce normal tubular architecture, and function. The return of glomerular filtration aids clearance of tubular debris and relief of obstruction. A period may exist where glomerular filtration has normalised, but tubular function remains deranged, hence the polyuric phase of ATN, where urine output is often excessive without normal homoeostasis.

The anuric phase of ATN classically lasts 7-21 days, and recovery to pre-insult levels of renal function can be expected, although some impairment of function may persist, particularly if there is a background of chronic renal insufficiency (see Outcomes section).

\section{CLINICAL APPROACH TO THE PATIENT}

The twin foundations of the approach to the patient with ARF are to:

(1) Treat any life threatening features.

Hypotension, shock and respiratory failure should be immediately apparent when assessing the patient, and clearly 


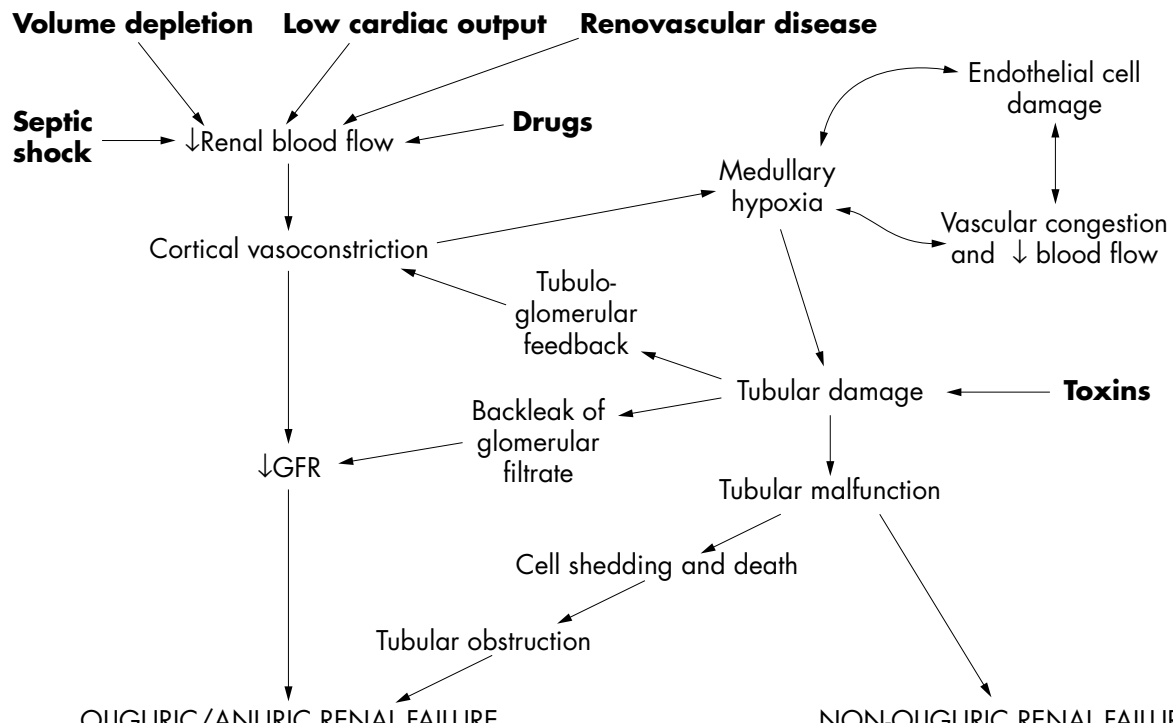

Figure 2 Mechanisms of acute tubular necrosis.

OLIGURIC/ANURIC RENAL FAILURE

these demand urgent treatment. Hyperkalaemia is less likely to be immediately obvious. Unless changes are evident on ECG or cardiac monitoring, it will only become apparent when chemistry is available.

(2) Identify any cause of ARF that warrants specific treatment

Many patients with ARF present with other diagnoses. Dehydration secondary to gastrointestinal losses, pneumonia, bowel obstruction, and new impairment of functional capacity in the elderly patient are often the initial "on take" diagnoses, and a diagnosis of renal failure is only made when laboratory parameters are available later. The clinician should then ask themselves the following questions (see box 1):

\section{(1) Is this acute or chronic renal failure?}

Previous laboratory data, information from case notes or GP may provide the answer, but in many cases such information will not be available. Anaemia, hypocalcaemia, and hyperphosphataemia are not good indicators of chronicity. Renal ultrasonography may show small $(<9 \mathrm{~cm})$ kidneys, often with cortical scarring and possibly cyst formation, in which case chronic disease is probable. The converse is not always true-the presence of two normal sized $(9-14 \mathrm{~cm}$ ) kidneys does not always indicate acute disease.

Box 1 History and examination in acute renal failure-general points

- Treat any life threatening features first-shock, respiratory failure, hyperkalaemia

- Is this acute or chronic renal impairment?

- A full drug history (current, recent, and alternative medication) is vital

- Is there a pre-renal cause? What is the patient's current fluid status?

- Could this be obstruction?

- Is intrinsic renal disease probable-what does urine analysis show?
(2) Are there other clues from the history?

Additional targeted history will often be necessary. Has there been a recent throat infection (possible post-streptococcal glomerulonephritis) and, if so, a course of amoxicillin (possible AIN)? A detailed drug history is important, and should include current and recent medications, and also over the counter preparations (especially NSAIDs), recreational drugs, and alternative or herbal remedies. Box 2 lists drugs commonly linked with AIN. ${ }^{9}$ Rash, fever, and arthralgia are also suggestive of AIN. Bone pain is a feature of myeloma.

Constitutional symptoms may point to systemic vasculitis. These frequently precede the acute presentation by many months, have often been dismissed by patients and clinicians alike (for example, nasal stuffiness and epistaxis as the initial symptoms of Wegener's granulomatosis), and only come to light during a detailed review of the history. Haemoptysis could show a pulmonary-renal syndrome.

In currently-or recently-hospitalised patients, nephrotoxic agents such as aminoglycosides and radiocontrast media must be rigorously excluded. After angiography renal function tends to reach its nadir a few days after the contrast dose. Cholesterol emboli may occur weeks or months later.

\section{(3) Is a pre-renal cause probable?}

Assessing the haemodynamic status of the patient is an essential part of the initial assessment and correction of hypovolaemia and hypotension may form part of the initial, lifesaving, management. Hypotension is usually easy to spot—but can be relative. A systolic pressure of $110 \mathrm{~mm} \mathrm{Hg}$ in the hypertensive patient whose normal value is around $160 \mathrm{~mm} \mathrm{Hg}$ can compromise renal perfusion. Postural hypotension (a decrease in systolic blood pressure of 20$30 \mathrm{~mm} \mathrm{Hg}$ from the lying to the upright position) and the jugular venous pressure (JVP), measured with the patient at $45^{\circ}$ to the horizontal, are invaluable markers of volume status. A significant postural decrease with the JVP not visible shows volume depletion and the need for fluid replacement, best carried out by rapid, repeated, infusions of small volumes $(250 \mathrm{ml})$, through secure venous access, with regular clinical reassessment. Once the patient is considered euvolaemic, replacement should be stopped or pulmonary oedema may occur. ${ }^{10}$ If fluid assessment is difficult ultrasound guided placement of an internal jugular catheter to measure central venous pressure may be necessary. The procedure is not without risks. These are 
Box 2 Common drug causes of acute interstitial nephritis

\section{Antimicrobial agents \\ - Penicillin \\ - Amoxycillin, \\ - Ampicillin \\ - Ciprofloxacin \\ - Rifampicin \\ - Sulphonamides \\ - Co-trimoxazole \\ - Aciclovir}

NSAIDs and salicylates

- Ibuprofen

- Naproxen

- Indomethacin

- Piroxicam

Anticonvulsants

- Phenytoin

Antiulcer agents

- Cimetidine

- Omeprazole

Others

- Thiazide diuretics

- Furosemide

- Allopurinol

- Mesalazine exacerbated in patients with ARF who may have collapsed veins, and increased risks of bleeding because of coagulopathy and platelet dysfunction. The time taken to perform the task must not delay fluid resuscitation. Evidence of current-or a history suggestive of recent-volume depletion implies a pre-renal cause or ATN, and response to treatment differentiates between the two.

\section{(4) Could this be obstruction?}

ARF is usually reversible with rapid relief of obstruction, but the longer the delay the more the long term damage. Obstruction should be sought by history and examination, before proceeding to urgent ultrasonography of the urinary tract. Prostatic disease is a common cause of ARF in men. Obstructive urinary symptoms may be elicited, a palpable bladder or hypertrophied prostate found on examination, and/or urethral catheterisation may yield a significant residual volume. In women, carcinoma of the cervix may be detectable on vaginal examination. There may be a history of renal stone disease. Obstruction can occur more proximally (fig 1).

Ultrasonography is an excellent tool for detecting obstruction; non-invasive, relatively simple, fast to perform, and portable. It also gives information on renal size. However, it is not perfect, and will fail to show hydronephrosis in about $5 \%$ of cases. Encasement of the renal pelvices and ureters by malignant tissue or retroperitoneal fibrosis may result in a failure to dilate even when obstructed, and dilatation may not be observed if there is an additional pre-renal element (with reduced GFR), immediately after acute obstruction ( $<24$ hours), or if the renal pelvis is abnormally small (an anatomical variant). The diagnosis should be entertained in those patients where obstruction seems likely on the basis of the clinical picture-computed tomography, cystoscopy with retrograde ureteropyelography and antegrade pyelography are alternative means of confirming the diagnosis. ${ }^{11}{ }^{12}$ Occasionally a trial of ureteric stenting or percutaneous nephrostomy is required.

\section{(5) Is intrinsic renal disease likely?}

We need to exclude renal inflammation that requires urgent diagnosis and treatment to prevent further loss-and aid recovery-of renal function. History (see section 2) and examination may provide clues (for example, vasculitic rash). Urine analysis is important and must be performed in all patients presenting with ARF. Haematuria or proteinuria suggest intrinsic renal disease. Urine should be microscoped and cultured. Red cell casts are highly suggestive of glomerulonephritis although only present in 30\% of cases. If intrinsic renal disease is considered probable, further urgent investigation is essential, as is urgent referral to a nephrologist-see later sections.

\section{(6) Initial investigations}

Initial investigations are aimed at identifying life threatening features, showing the severity of renal failure and any comorbidity, and attempting to identify the cause. There are a number of common investigations, then those targeted at specific disease entities-see box 3 and table 1 .

The relative increase in urea and creatinine can provide clues to the cause of renal impairment: as a rough guide the blood urea $(\mathrm{mmol} / \mathrm{l})$ should be $10 \%$ of the serum creatinine $(\mu \mathrm{mol} / \mathrm{l})$. Volume depletion causes a greater increase in urea relative to creatinine, as does gastrointestinal blood loss. A comparatively low blood urea may occur in severe liver disease. Muscle necrosis may cause an inappropriately high serum creatinine. The assessment of urine osmolality and sodium concentration, and calculation of fractional excretion of sodium, has previously been advocated to permit distinction between pre-renal failure and ATN. In practice, however, this is rarely useful; clinical features and response to treatment (mainly fluid challenge) are much more significant. Hypercalcaemia, a high erythrocyte sedimentation rate (ESR), and raised total protein levels may be indicative of myeloma.

Renal biopsy may be indicated when intrinsic renal disease is suspected, especially if serology is non-diagnostic.

\section{Box 3 Common investigations: all cases of ARF}

- Urine analysis

- Urine specimen for microscopy and culture

- Basic bloods:

- Urea and electrolytes

- Full blood count

- Blood glucose

- Coagulation screen

- Inflammatory markers

- Liver function tests

- Calcium and phosphate

- Blood cultures (if infection suspected)

- Arterial blood gases or venous bicarbonate

- Electrocardiogram

- Chest radiograph

- Renal ultrasonography 
Table 1 Specific investigations in ARF

\begin{tabular}{ll}
\hline Indication/potential cause & Investigation \\
\hline $\begin{array}{l}\text { Intrinsic renal disease } \\
\text { (glomerulonephritis or AIN) }\end{array}$ & $\begin{array}{l}\text { Autoimmune serology-ANCA, ANA, } \\
\text { anti-GBM } \\
\text { Complement levels (C3, C4) } \\
\text { Consider cryoglobulins }\end{array}$ \\
$\begin{array}{l}\text { Post-streptococcal } \\
\text { glomerulonephritis }\end{array}$ & $\begin{array}{l}\text { (ASOT) } \\
\text { Possible myeloma }\end{array}$ \\
$\begin{array}{l}\text { Serum immunoglobulin titres and } \\
\text { paraprotein electrophoresis, urinary } \\
\text { Bence-Jone protein }\end{array}$ \\
$\begin{array}{l}\text { Rhabdomyolysis } \\
\text { Renal stone disease }\end{array}$ \\
$\begin{array}{l}\text { Plain abdominal radiograph, } \\
\text { abdominal computed tomography } \\
\text { for dialysis }\end{array}$ & $\begin{array}{l}\text { Hepatitis B and C serology (plus } \\
\text { consider HIV testing) }\end{array}$ \\
\hline ANCA, antineutrophil cytoplasmic antibody; ANA, antinuclear antibody; \\
anti-GBM, antiglomerular basement membrane antibody.
\end{tabular}

Consideration of renal biopsy should be undertaken in conjunction with a nephrologist.

\section{PRACTICAL MANAGEMENT}

Management is directed at treating any life threatening features, attempting to halt or reverse the decline in renal function, and if unsuccessful providing support by renal replacement anticipating renal recovery. Hyperkalaemia, pulmonary oedema, and severe acidosis require immediate attention. Fluid balance, the treatment of less severe acidosis, the use of diuretics and dopamine, as well as the relief of obstruction are all issues in the further management of the patient-some more controversial than others. Provided the patient can be maintained through the period of nonfunction, and no further insults accrue, the kidney is remarkable in its ability to recover its normal homoeostatic role.

\section{(1) Hyperkalaemia}

Severe hyperkalaemia (plasma potassium $\left([\mathrm{K}+]_{\mathrm{p}}\right)$ $>6.5 \mathrm{mmol} / \mathrm{l}$ ) is a medical emergency because of the risk of life threatening cardiac arrhythmias. Figure 3 gives the causes of hyperkalaemia in ARF. As $[\mathrm{K}+]_{\mathrm{p}}$ rises, a typical pattern of ECG changes ensues: peaked/tented $\mathrm{T}$ waves $\left([\mathrm{K}+]_{\mathrm{p}}>6.5 \mathrm{mmol} / \mathrm{l}\right)$; flattening of the $\mathrm{P}$ wave and prolongation of the QRS complex $\left([\mathrm{K}+]_{\mathrm{p}} 7-8 \mathrm{mmol} / \mathrm{l}\right)$; sine waves $\left([\mathrm{K}+]_{\mathrm{p}} 8-9 \mathrm{mmol} / \mathrm{l}\right)$; and ventricular fibrillation or asystole $\left([\mathrm{K}+]_{\mathrm{p}}>9 \mathrm{mmol} / \mathrm{l}\right)^{13}$ (fig 4$)$. These changes are not always consistent, however, and patients with a normal baseline ECG may also develop arrhythmias. Urgent treatment of hyperkalaemia should be started if the serum potassium is $>6.5 \mathrm{mmol} / \mathrm{l}$, or if any ECG changes are present.

The treatment of hyperkalaemia can be divided into four steps (table 2)

\section{Stabilisation of cardiac myocyte}

Calcium antagonises the effects of hyperkalaemia, stabilising the myocardium within a few minutes of infusion and producing a more normal ECG trace without affecting serum potassium. It should be given immediately if $\mathrm{P}$ wave or QRS changes are present. A bolus of $10-20 \mathrm{ml}$ of $10 \%$ calcium gluconate or chloride is given intravenously over two to five minutes-the former is preferable because of a lower risk of tissue damage if extravasation occurs. High serum calcium concentrations potentiate the cardiac toxicity of digoxincalcium should be given as a slow infusion in hyperkalaemic patients taking this drug (that is, $10 \mathrm{ml} 10 \%$ calcium gluconate in $100 \mathrm{ml} 5 \%$ dextrose over 30 minutes). ${ }^{14}$
Reduction in plasma potassium concentration

Treatment with calcium is a temporising measure "buying time" while measures are started to reduce the serum potassium through increasing cellular uptake. Various options exist:

\section{Insulin with glucose}

Insulin acts rapidly to indirectly activate the cell membrane $\mathrm{Na}+/ \mathrm{K}+-$ ATPase and thus increase cellular potassium uptake, probably via activation of $\mathrm{Na}+\mathrm{H}+$ channels and an increase in intracellular $[\mathrm{Na}+] .{ }^{15}$ The addition of glucose to the insulin bolus is necessary to prevent hypoglycaemia. Ten units of fast acting soluble insulin should be added to $50 \mathrm{ml}$ of 50\% dextrose and infused over 10-20 minutes. A reduction in $[\mathrm{K}+]_{\mathrm{p}}$ is seen after $20-30$ minutes. Insulin alone can be given to hyperglycaemic patients (blood glucose $>14 \mathrm{mmol} / \mathrm{l}$ ) as the infusion of further glucose can worsen hyperkalaemia secondary to its osmotic effect. ${ }^{16}$ This, plus the need for rapidly attained supraphysiological insulin levels to produce a hypokalaemic effect, explain the inadequacy of glucose infusion alone as treatment for hyperkalaemia in nondiabetic patients. ${ }^{17}$ Whether insulin and dextrose, or insulin alone, is used, the blood glucose should be monitored carefully for at least six hours. Hypoglycaemia occurred in up to $75 \%$ of patients in some studies, and was generally associated with higher insulin or lower glucose doses. ${ }^{13}$

\section{$\beta_{2}$ adrenergic agonists}

Salbutamol binds to $\beta_{2}$ receptors and through cytosolic second messengers activates the $\mathrm{Na}+\mathrm{K}+-$ ATPase, thus promoting cellular potassium uptake. Nebulised and intravenous salbutamol produce a similar effect to insulin, but at higher doses than used for bronchospasm (10-20 mg via nebuliser, or $0.5 \mathrm{mg}$ intravenously). Up to $40 \%$ of patients do not respond. Tachycardia is common especially after intravenous administration. The method should not be used in patients taking $\beta$ blockers or in those with a high risk of cardiac side effects. For these reasons, insulin is the agent of choice to reduce $[\mathrm{K}+]_{\mathrm{p}}$, but salbutamol may be preferably in certain circumstances, especially paediatric patients, ${ }^{18}$ and combined therapy with insulin and dextrose plus salbutamol may be more effective than either treatment alone. ${ }^{13}$

\section{Sodium bicarbonate}

The infusion of sodium bicarbonate has little immediate effect on hyperkalaemia, but may be used to correct acidosis (see later).

\section{Removal of potassium from the body}

While the above techniques serve to redistribute potassium, the overall amount retained by a patient with ARF will still be excessive. If purely pre-renal failure is present, and responds to resuscitation, then increased renal potassium excretion will serve to return whole body levels to normal. Additional means of increasing potassium loss are often needed in those whose renal function fails to improve.

\section{lon exchange resins}

These bind potassium in the gastrointestinal tract, in exchange for calcium or sodium, and result in increased potassium excretion in the stool. Calcium resonium (calcium polystyrene sulphonate) and Resonium A (sodium polystyrene sulphate) are the most commonly used, given at an oral dose of $15 \mathrm{~g}$ up to thrice daily, together with an osmotic laxative (for example, lactulose $10 \mathrm{ml}$ ) to prevent constipation. They can also be given rectally. An effect takes two to three hours. Side effects include hypercalcaemia and salt/ water overload (with calcium and sodium containing resins respectively), and hypomagnesaemia. They are most suited for use in situations where potassium levels are moderately 


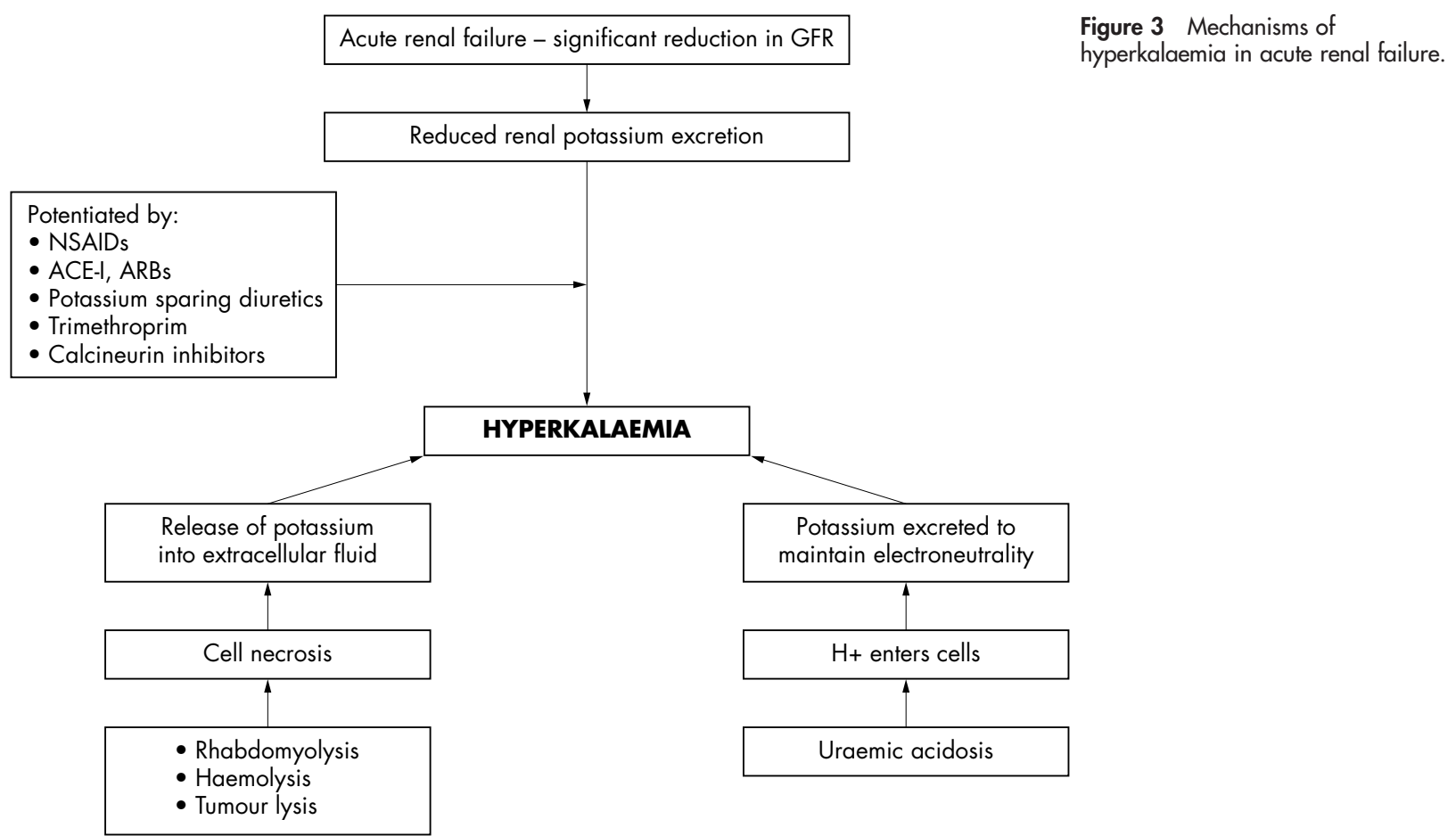

high (around $6.5 \mathrm{mmol} / \mathrm{l}$ ), comparatively stable, and improvement in the patient's renal function is expected or dialysis is planned but can be delayed.

\section{Haemodialysis}

This is the definitive means by which potassium can be removed from the body, and is indicated in refractory severe hyperkalaemia. Haemodialysis is more effective than haemofiltration or peritoneal dialysis at potassium removal and has an immediate effect once started. Maximum removal occurs in the first hour of dialysis. Serum potassium may decrease by $1.2-1.5 \mathrm{mEq} / \mathrm{l} / \mathrm{h}$ if potassium free dialysate is used, which can precipitate hypokalaemia and arrhythmias. Generally a dialysate potassium concentration of 1.0 or $1.5 \mathrm{mmol} / \mathrm{l}$ is used. Higher concentrations may be used in those at risk of arrhythmias (especially if taking digoxin), and a lower concentration where there is a continued hyperkalaemic drive such as rhabdomyolysis, tumour lysis, and haemolysis. Some rebound of potassium levels occurs after dialysis, especially if initial levels were high or if treatments to increase cellular uptake were used pre-dialysis ${ }^{14-16}$

Prevention of further potassium accumulation. This can be achieved through a low potassium diet.

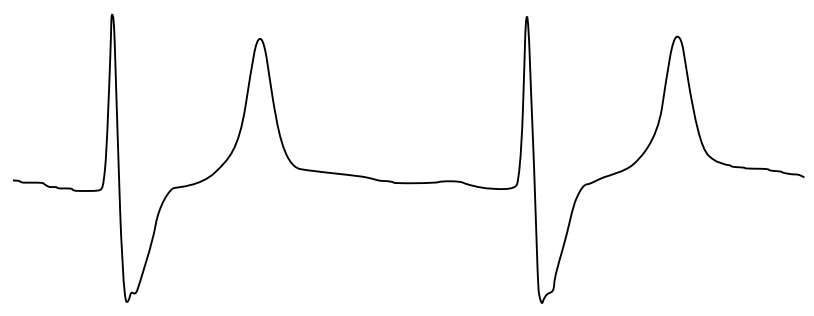

Figure 4 An ECG from a haemodialysis patient with a serum potassium of $8.0 \mathrm{mmol} / \mathrm{l}$, showing the classic changes of significant hyperkalaemia: tented T waves, flattening of the $\mathrm{P}$ wave, and prolongation of the QRS complex.

\section{(2) Pulmonary oedema}

The oligo-anuric patient with pulmonary oedema resulting from fluid overload (with/without underlying cardiac disease) represents a clinical challenge. As previously mentioned, if significant ventilatory failure is present, this must be dealt with first, through supplementary oxygen, noninvasive ventilation, or intubation and ventilation, depending on the state of the patient. While these measures are being undertaken, pharmacological treatment to offload the decompensated heart can be started-intravenous opioids (diamorphine $2.5-5 \mathrm{mg}$, with care taken depending on the degree of respiratory distress) and an intravenous infusion of nitrate (for example, glyceryl trinitrate $50 \mathrm{mg}$ in $50 \mathrm{ml} 0.9 \%$ saline, at a rate of $2-20 \mathrm{ml} / \mathrm{h}$ keeping the systolic blood pressure $>95 \mathrm{~mm} \mathrm{Hg}$ - and attempts made to provoke a diuresis. Much larger doses of diuretics are required in renal failure, for example, furosemide $250 \mathrm{mg}$ in $50 \mathrm{ml} 0.9 \%$ saline over one hour, with an effect seen within one to two hours, if at all, and which can be repeated if effective. Venesection is another desperate measure.

If these interventions are not successful, or if the patient is in extremis such that they seem unlikely to prove effective, then fluid removal by renal replacement therapy is the definitive answer. Either haemodialysis or haemofiltration can be used, the choice of modality often determined by the additional needs of the patient, particularly the need for cardiovascular and respiratory support, and the location in which they can be best served.

Pulmonary oedema is often the result of excessive fluid resuscitation, and can be anticipated in many patientsespecially those with known cardiac dysfunction, the elderly, and those who appear volume replete at the outset-and hopefully avoided by more judicious intravenous fluid therapy (see section 4 below).

\section{(3) Acidosis}

Severe metabolic acidosis (blood $\mathrm{pH}<7.2$ ) often accompanies ARF and arises through a variety of mechanisms, related both to reduced renal function and the underlying cause of the patient's illness. Systemic acidosis impairs cardiac 
Table 2 Summary of treatment strategies in hyperkalaemia (see text for references)

\begin{tabular}{|c|c|c|c|}
\hline Treatment & Immediacy of action & Reduction in $[\mathbf{K}+]_{\mathbf{p}}$ & Duration of action \\
\hline $\begin{array}{l}\text { Calcium gluconate/ } \\
\text { carbonate }\end{array}$ & $1-3 \min$ & Nil & $30-60 \mathrm{~min}$ \\
\hline Insulin (+/- dextrose) & $15-30 \mathrm{~min}$ & $0.65-1.0 \mathrm{mmol} / \mathrm{l}$ & $4-6 h$ \\
\hline $\begin{array}{l}\text { Salbutamol (nebulised/ } \\
\text { intravenous) }\end{array}$ & $30 \mathrm{~min}^{*}$ & $0.6-1.0 \mathrm{mmol} / \mathrm{I}$ & $2-4 \mathrm{~h}$ \\
\hline $\begin{array}{l}\text { lon exchange resin } \\
\text { Haemodialysis }\end{array}$ & $\begin{array}{l}2-3 \mathrm{~h} \\
\text { Immediate }\end{array}$ & $\begin{array}{l}0.5-1.0 \mathrm{mmol} / 1 \mathrm{~g} \text { resin } \\
\leqslant 1.5 \mathrm{mmol} / \mathrm{l} / \mathrm{h}\end{array}$ & $\begin{array}{l}4-6 \mathrm{~h} \\
\text { While dialysis ongoing }\end{array}$ \\
\hline
\end{tabular}

contractility, induces bradycardia, produces vasodilatation, and augments hyperkalaemia, among other effects. Reversing acidosis through administration of an alkaline solutionsodium bicarbonate-would seem to be sensible, but there is very little evidence to show that it provides benefit. Bolus therapy with hypertonic (8.4\%) sodium bicarbonate can worsen intracellular acidosis (while deceiving the clinician through improving blood $\mathrm{pH}$-paradoxical acidosis) and cause osmotic vasodilatation, overshoot metabolic alkalosis, hypernatraemia, and extravasation injury if not delivered into a central vein. ${ }^{19}$ Both $8.4 \%$ and $1.26 \%$ solutions provide a sodium load, which causes extracellular fluid volume expansion and can push an already volume overloaded patient into pulmonary oedema. Isotonic $(1.26 \%)$ solutions may have a role as fluid replacement therapy in stable patients with a moderate to severe acidosis and a requirement for fluid replacement, in whom dialysis is not imminent. Haemodialysis or haemofiltration will usually be required to treat severe acidosis in oligoanuric patients.

\section{(4) Fluid balance}

The approach to the hypovolaemic patient with pre-renal failure has been described earlier. Which is the optimal resuscitation fluid is still debated. Normal saline and $4 \%$ albumin have clinical equivalence, shown by the recent SAFE study of patients admitted to intensive care units in Australia and New Zealand, and which overturns previous studies showing that albumin use may be associated with a higher mortality. ${ }^{20} 21$ Once the patient is considered euvolaemic (normotensive, no postural drop, JVP and/or central venous pressure (CVP) normal), care should be taken to avoid fluid overload and a maintenance regimen started, which takes account of renal and insensible losses, aiming for a positive balance of $500 \mathrm{ml} /$ day (hourly input = previous hour's output plus $25 \mathrm{ml}$ ). This requires accurate observation and record keeping by nursing staff, and regular re-assessment by the clinician.

\section{(5) Dopamine}

The use of low dose $(1-3 \mu \mathrm{g} / \mathrm{kg} / \mathrm{min})$ dopamine has been advocated to increase renal perfusion in critically ill patients. Recent studies, including a large randomised controlled trial, have shown it to lack efficacy on renal outcome or overall mortality. ${ }^{22}{ }^{23}$ Use of dopamine may also reduce splanchnic perfusion, depress respiration, suppress anterior pituitary hormone release and function, and worsen renal function in hypovolaemic or normovolaemic patients. ${ }^{23}$ Its routine use in ARF is not currently justifiable. ${ }^{24}$

\section{(6) Diuretics}

There is a theoretical rationale for the use of loop diuretics in ARF-inhibition of the $\mathrm{Na}+/ \mathrm{K}+/ 2 \mathrm{Cl}-$ pump in the thick ascending limb of the loop of Henle, with subsequent decrease in $\mathrm{Na}+\mathrm{K}+-$ ATPase activity, should reduce the oxygen requirements of these cells, and thus their susceptibility to ischaemic damage. ${ }^{25}$ There are scarce clinical data to support this, and recent studies have either correlated the use of diuretics with increased mortality, ${ }^{26}$ or shown no benefit. ${ }^{27}$ It seems reasonable to use diuretics only in adequately resuscitated-but oliguric - patients, at a dose suitable to the degree of renal impairment $(250 \mathrm{mg}$ furosemide intravenously over one hour is a standard regimen), and to stop diuretic treatment if oliguria persists. Converting oliguric to non-oliguric renal failure may help with fluid and electrolyte management, but does not seem to affect eventual need for dialysis or overall mortality, and should not delay the start of renal replacement therapy when otherwise indicated.$^{28}$ There are no data to support the use of mannitol.

\section{(7) Relief of obstruction}

It is important to relieve urinary tract obstruction promptly. Bladder outflow obstruction can be relieved by passage of a urethral catheter-which should be considered in all patients with ARF to accurately measure urine output-but relief of upper tract obstruction may require either antegrade (percutaneous nephrostomy) or retrograde (cystoscopy and retrograde ureteral catheterisation) approaches. Urethral catheterisation can be performed immediately, but other techniques require planning. Close collaboration between nephrological, urological, and radiological services is required, and in many cases renal replacement therapy may be necessary before relief of obstruction can be achieved.

A significant diuresis can complicate relief of complete urinary tract obstruction, through both appropriate (excretion of retained solute and water) and inappropriate mechanisms (tubular concentrating dysfunction). Severe polyuria is rare and requires careful management to prevent volume depletion and possible pre-renal impairment, or overzealous fluid resuscitation and a further drive to diuresis. ${ }^{29}$ Obstruction may result in an impaired distal tubular response to aldosterone, resulting in a paradoxical hyperkalaemic acidosis when relieved. This usually resolves spontaneously. A small number will have permanent tubular damage and a persistent salt wasting nephropathy. ${ }^{30} 31$

\section{(8) General measures}

In addition to the specific measures discussed above, the ongoing care of the patient with ARF should be directed at preventing further renal insults and supporting normal physiology. Additional renal damage is often accrued through recurrent episodes of reduced renal perfusion, sometimes difficult to avoid in the unstable patient, but also through the administration of nephrotoxic drugs, most often NSAIDs, ACE-I, and aminoglycoside and glycopeptide antibiotics. The latter two should be dosed according to monitored plasma concentrations. There are many other drugs that require dose adjustment depending on the patient's GFR, not because they are directly nephrotoxic, but because impaired renal excretion changes their pharmacodynamics (for example, cephalosporin antibiotics, morphine). The use of contrast media should also be avoided (see later).

Patients with ARF may suffer from excessive bleeding, because of uraemia induced platelet dysfunction and coagulopathies (for example, sepsis associated disseminated 
intravascular coagulation). Correction of coagulopathy may be necessary with blood products and vitamin K. Renal replacement therapy (RRT) may improve platelet function but DDAVP and cryoprecipitate may be required. ARF is associated with numerous metabolic disturbances but energy expenditure is not increased significantly. ${ }^{32}$ Associated conditions such as sepsis and burns, however, often lead to hypercatabolic states. Carbohydrate and protein requirements should be tailored individually and ideally delivered via the enteral route. Parenteral administration may be necessary in some. Volume is an important consideration and needs to be factored into RRT prescription. Additional water soluble vitamins may be required, as these are removed by RRT. $^{32}{ }^{33}$ There is an increased susceptibility to infection. Good infection control practices and a low threshold for considering an infectious aetiology for any clinical deterioration may minimise the risk. Care of pressure areas and prophylaxis against deep venous thrombosis can avoid some of the problems of prolonged immobility.

\section{INDICATIONS FOR RENAL REPLACEMENT THERAPY}

Some patients warrant nephrological or intensive care support from the outset, some respond to the medical measures outlined above and recover, and others may require nephrological input later in the course of their illness. As a broad generalisation, patients with ARF as part of a systemic illness-often with failure of multiple organ systemsrequire intensive care unit admission, given their probable needs for intensive monitoring, nursing and support of other organ systems. Patients with single organ renal failure can usually be managed on a renal ward, and nephrologists will often support the role of the intensivist on the intensive care unit. The severity of the ARF will determine the urgency for nephrology referral but this should be the same day if intrinsic renal disease is suspected.

Guidelines for the start of RRT are given in box $4 .{ }^{34}$ It must be emphasised that the patient should be viewed as a whole, and dialysis neither started nor withheld on the basis of a single variable. It is common practice to start RRT at an "earlier" stage in patients with multiorgan failure because of their potential for further deterioration and the benefits that RRT may bring. Before starting RRT, it must also be considered whether aggressive treatment is in the patient's best interests, especially in those with significant pre-morbid functional impairment and extra-renal comorbidity. The patient and their family should—wherever possible—be part of this process.

\section{CHOICE OF RRT MODALITY}

RRT can be intermittent or continuous. Continuous dialysis can be blood based or use the peritoneal route. However, peritoneal dialysis is now rarely used in the treatment of ARF in developed

Box 4 Who needs dialysis? Guidelines for the initiation of renal replacement therapy

- Severe hyperkalaemia, unresponsive to medical therapy

- Fluid overload with pulmonary oedema (in the context of acute renal failure)

- Uraemia (blood urea $>30-50 \mathrm{mmol} / \mathrm{l}$ )

- Complications of severe uraemia: encephalopathy, pericarditis, neuropathy/myopathy

- Severe acidosis ( $\mathrm{pH}<7.1$ )

- Drug overdose with a dialysable toxin countries. It may still have a role where anticoagulation is not possible, vascular access not feasible, or when very gentle fluid removal is required, and where the resources for blood based approaches do not exist. Outcomes are poorer with peritoneal dialysis than with continuous haemofiltration in the treatment of infection associated ARF. ${ }^{35}$

Intermittent therapy is delivered as intermittent haemodialysis (IHD), and continuous renal replacement therapy (CRRT) in a number of ways, classified according to the method of accessing the circulation and how solute removal is achieved. Techniques based on venous access alone are most frequently used-continuous venovenous haemofiltration (CVVH) and continuous venovenous haemodiafiltration (CVVHD) - the latter including solute removal by diffusion (haemodialysis) in addition to fluid and solute removal by convection (haemofiltration). A full discussion of CRRT methods is beyond the scope of this article but there are a number of excellent published reviews. ${ }^{36}{ }^{37}$ Table 3 gives the advantages and disadvantages of continuous compared with intermittent strategies (adapted from Lameire et $a l^{30}$ ). There is no firm evidence that outcomes are any different with continuous or intermittent therapy, ${ }^{38}$ but in those patients treated with haemodialysis survival is improved through the use of daily treatment. ${ }^{39}$ The benefits of biocompatible membranes are still contentious. ${ }^{40}{ }^{41}$ Adopting a common sense approach, CRRT is preferable for the patient with multiple organ dysfunction and cardiovascular instability, possibly with significant fluid overload, and IHD for the stable patient with single organ failure. This mirrors our earlier recommendations on where such patients should be managed, sicker patients benefiting more from the increased monitoring facilities and higher levels of nursing support provided by an intensive care unit. A newer technique, slow low efficiency dialysis (SLED), which uses extended haemodialysis treatment sessions with gradual fluid removal, may offer the advantages of intermittent therapies with the haemodynamic stability and metabolic control associated with CRRT. ${ }^{42} 43$

\section{SPECIAL SITUATIONS}

\section{(1) Rapidly progressive glomerulonephritis}

Rapidly progressive glomerulonephritis (RPGN) can destroy kidney function within days to weeks if not correctly identified and treated. Pauci-immune RPGN producing ARF is often a feature of ANCA associated vasculitis, although renal limited disease and ANCA negative disease are also fairly common. Other immune conditions cause an RPGN but are less common. These include antiglomerular basement membrane antibody (Goodpasture's) disease, HenochSchonlein disease, systemic lupus erythematosus (SLE), cryoglobulinaemias, post-infectious nephritis, and crescentic change in primary glomerular disease such as mesangiocapillary glomerulonephritis. Clinical suspicion, active urinary deposit, serology, and renal biopsy are the keys to diagnosis. The nephrologist should be involved as early as possible if RPGN is considered. Once a diagnosis has been made, specific therapy is necessary-immune suppression with corticosteroids and other cytotoxics (usually cyclophosphamide), plus plasma exchange in some circumstances. ${ }^{44}$

\section{(2) Haemolytic-uraemic syndrome}

This occurs in an epidemic form, usually associated with outbreaks of diarrhoea often caused by Shiga toxin producing $E$ coli O157:H7 (D+), and sporadic forms (D-). Diagnosis rests on clinical suspicion and haematological findings including red cell fragments on blood film. Urgent nephrological referral is required. Treatment of $\mathrm{D}+$ disease is supportive. Plasma exchange with fresh frozen plasma may benefit $\mathrm{D}-$ disease, and tight control of blood pressure is vital. 


\begin{tabular}{|c|c|c|}
\hline & Intermittent haemodialysis & Continuous therapy \\
\hline Advantages & $\begin{array}{l}\text { Lower risk of systemic bleeding } \\
\text { More time available for diagnostic and } \\
\text { therapeutic interventions } \\
\text { More suitable for severe hyperkalaemia } \\
\text { Lower cost } \\
\text { Fewer cardiac arrhythmias } \\
\text { Shorter stay in ICU }\end{array}$ & $\begin{array}{l}\text { Better haemodynamic stability } \\
\text { Better fluid control } \\
\text { Better biochemical control } \\
\text { Better pulmonary gas exchange } \\
\text { Improved nutritional support }\end{array}$ \\
\hline Disadvantages & $\begin{array}{l}\text { More difficult haemodynamic control } \\
\text { Availability of dialysis staff } \\
\text { Inadequate dialysis dose } \\
\text { Inadequate fluid control } \\
\text { Not suitable for patients with intracranial } \\
\text { hypertension } \\
\text { No removal of cytokines }\end{array}$ & $\begin{array}{l}\text { Higher risk of systemic bleeding } \\
\text { Greater vascular access problems } \\
\text { More filter problems } \\
\text { More immobilisation of patient } \\
\text { Greater cost }\end{array}$ \\
\hline
\end{tabular}

\section{(3) Tubulointerstitial nephritis}

Drug induced tubulointerstitial nephritis should respond to cessation of the offending agent but renal biopsy may be required to confirm the diagnosis, and exclude other pathology. Nephrological referral is required. Corticosteroids are often used to retard the inflammatory response and aid recovery of renal function, but evidence for their efficacy remains debatable.

\section{(4) Contrast nephropathy}

The use of radiological contrast media is associated with the development of ARF in a number of at risk groups including patients with pre-existing renal impairment (especially diabetic nephropathy), those with volume depletion, those taking drugs that interfere with the normal regulation of renal perfusion (ACE-I, NSAIDs), and those requiring increased doses of contrast agent. ${ }^{45}$ Peak creatinine levels are normally reached at three to five days, usually returning to baseline within another five days, but more severe damage can occur in high risk groups. The incidence of this complication in at risk patients can be minimised by:

- Pre-hydration with normal saline (hydration with sodium bicarbonate may prove more efficacious but further data are required ${ }^{46}$ ).

- Treatment with $\mathrm{N}$-acetyl cysteine (NAC)-600 mg orally twice daily on the day before and the day of the procedure ${ }^{45}$ The evidence is conflicting, but this is a low cost, easily given, widely available drug with minimal side effects.

- The use of the lowest possible volume of iso-osmolar contrast. ${ }^{47} 48$

- Temporary cessation of other nephrotoxic drugs periprocedure.

\section{(5) Rhabdomyolysis}

Severe skeletal muscle injury and cell lysis produces rhabdomyolysis with the release of muscle breakdown products into the circulation. ARF may result, partly from direct toxic effects of myoglobin, and partly from intravascular volume depletion (muscle oedema, plus blood loss from trauma) and renal hypoperfusion. Rhabdomyolysis is a comparatively common cause of ARF, and causes range from traumatic crush injuries to drug side effects. ${ }^{49}$ It is important to consider the diagnosis at an early stage-and confirm it with measurement of muscle creatinine kinase-because correct treatment may prevent established renal failure. Patients with rhabdomyolysis should be aggressively fluid resuscitated with saline to achieve high urinary flow rates (urine output $>200 \mathrm{ml} / \mathrm{h}$ ). ${ }^{50}$ Sodium bicarbonate and mannitol have been used to correct acidosis and force diuresis respectively, but evidence of efficacy is lacking. ${ }^{51}$ Nevertheless, the use of isotonic sodium bicarbonate in the acidotic, hyperkalaemic patient who requires fluid replacement seems reasonable..$^{50}$

\section{(6) Liver disease-hepatorenal syndrome}

Hepatorenal syndrome (HRS) defines the ARF that occurs in conjunction with advanced cirrhotic liver disease when other causes of renal impairment such as pre-renal failure, ATN, and AIN have been excluded (not always simple). It is subdivided into types-(1) rapidly progressive renal impairment, which presents as ARF and (2) slowly progressive or stable renal impairment, which presents with recurrent or refractory ascites poorly responsive to diuretics. The outcome of type l HRS is extremely poor, with survival to hospital discharge less than $10 \%$. Liver transplantation, when indicated, remains the best therapeutic option. Survival may be improved with vasoconstrictors (for example, terlipressin) in conjunction with albumin, which produce splanchnic vasoconstriction and improve renal perfusion, or with a transjugular intrahepatic portosystemic shunt (TIPS). RRT is usually only started if liver transplantation is considered a viable option. ${ }^{52}$

\section{NOVEL THERAPIES}

Early goal directed therapy aiming to optimise renal (and other organ) perfusion shows some success in the treatment of ARF, and tight control of blood glucose reduces the need for RRT. ${ }^{53}$ More specific experimental treatments include antiendothelin antibodies, oxygen free radical scavengers, and inhibitors of inducible nitric oxide synthetase, all designed to reduce renal damage occurring in the context of sepsis. Infusions of atrial natriuretic peptide (ANP), or a synthetic analogue anaritide, may improve renal perfusion, ${ }^{54}$ and recombinant erythropoietin can reduce ischaemic renal injury in an animal model. ${ }^{55}$ Therapeutic strategies in the more distant future may include bioartificial kidneys as a renal replacement modality - the combination of a haemofilter plus renal tubular cells, designed to reduce circulating pro-inflammatory mediators-and possible stem cell therapy to improve native kidney recovery. ${ }^{56}$

\section{OUTCOMES}

Despite our best therapeutic efforts, ARF is a serious condition that carries a considerable mortality, about $40 \%-$ $70 \%$ depending on the population studied and definitions used. ${ }^{1}{ }^{27-62}$ This mortality rate has remained constant over the past 40 years, perhaps because the lower thresholds for 


\section{Box 5 Prevention of acute renal failure}

- Recognise that patients with pre-existing renal impairment are at higher risk of developing further renal insufficiency - treat and monitor accordingly

- Temporarily withhold nephrotoxics (especially ACE-I, ARBs, NSAIDs) and diuretics (to prevent dehydration) when patients become unwell-either in the community or in hospital

- Ensure that patients remain adequately hydrated, to maintain renal perfusion

- Remember to monitor renal function after starting, or increasing the dose, of ACE-I or ARBs (check one to two weeks later)

- Where necessary, adjust drug doses in patients with renal impairment

- Monitor drug levels when using aminoglycoside (gentamicin) and/or glycopeptide (vancomycin) antibiotics - and adjust dose accordingly

- Hydrate the patient and consider using $\mathrm{N}$-acetyl cysteine before procedures entailing radiological contrast media

high risk diagnostic and therapeutic interventions in those with increased comorbidity, and the reduction in the numbers of patients with "simple" ARF (for example, related to obstetric complications), have negated the effect of therapeutic advances on prognosis. ${ }^{306}$ Immediate prognosis is worse with increasing age and comorbidity. A significant proportion fail to recover renal function and require long term renal replacement therapy (up to $17 \%$ of survivors in one study) - this proportion is much higher in those with pre-existing renal impairment..$^{57}{ }^{58}$ Recovery of renal function is often incomplete and chronic renal failure may result, with its associated increased cardiovascular mortality and attendant risks of later progression to end stage disease. ${ }^{2}$

As physicians, we should pay more attention to the prevention of $\mathrm{ARF}$, recognising that our sick patients are at high risk of developing renal impairment, and seeking to avoid perturbations in cardiovascular status, the prescription of nephrotoxic drugs, and use of procedures that increase the risk (box 5). Once ARF is established, a precise diagnosis should be sought and appropriately treated, and further insults avoided. The cornerstone of effective management remains regular reassessment of the patient's clinical and biochemical status, and recognition of the need for additional intervention, including timely and effective renal replacement therapy.

\section{MULTIPLE CHOICE QUESTIONS (TRUE (T)/FALSE (F)); ANSWERS AT END OF REFERENCES}

1. Acute renal failure:

(A) Affects about 5\% of hospital admissions

(B) Decreases in frequency with increasing age

(C) Has a mortality rate of about $50 \%$

(D) Results in a need for continued renal replacement therapy in $50 \%$ of cases

(E) Is usually attributable to intrinsic renal disease

2. When faced with a patient with renal impairment:

(A) Anaemia is a good indicator of chronicity

(B) Thiazide diuretics, amoxicillin and ibuprofen are all common causes of acute interstitial nephritis

\section{Five key references}

- Vinen CS, Oliveira DBG. Acute glomerulonephritis. Postgrad Med J 2003;79:206-13.

- Glynne P, Allen A, Pusey C. Acute renal failure in practice. London: Imperial College Press, 2002.

- Lameire N, Van Biesen W, Vanholder R. Acute renal failure. Lancet 2005;365:417-30.

- Schrier R, Wei Wang. Mechanisms of disease: acute renal failure and sepsis. N Engl J Med 2004;315:15969.

- Asif A, Epstein M. Prevention of radiocontrast-induced nephropathy. Am J Kidney Dis 2004;44:12-24.

(C) Renal ultrasonography may fail to detect obstruction

(D) Red cell casts are present in the urine of almost all patients with glomerulonephritis

(E) Rhabdomyolysis produces an inappropriately low serum creatinine

3. In patients with hyperkalaemia:

(A) Peaked/tented $\mathrm{T}$ waves occur with a plasma $[\mathrm{K}+]$ $\geqslant 5.5 \mathrm{mmol} / \mathrm{l}$

(B) Double the amount of intravenous calcium should be given in patients also taking digoxin

(C) A much higher dose of salbutamol must be used than when treating bronchospasm

(D) Ion exchange resins may produce hypermagnesaemia

(E) Haemodialysis is indicated in refractory hyperkalaemia

4. When treating patients with ARF:

(A) The use of bicarbonate solutions to treat acidosis may be detrimental

(B) The use of low dose dopamine to increase renal perfusion should remain standard therapy

(C) Diuretics have been shown to lessen mortality

(D) The SAFE study showed poorer outcomes when patients were treated with albumin (rather than crystalloid)

(E) Renal biopsy is essential in all cases of established ARF

5. In patients with acute renal failure:

(A) Peritoneal dialysis is the treatment of choice in patients with cardiac dysfunction

(B) Continuous venovenous haemofiltration confers a survival advantage over intermittent haemodialysis

(C) In inotrope dependent patients continuous venovenous haemofiltration is preferable to intermittent haemodialysis

(D) Renal replacement therapy is normally not indicated until the serum creatinine is over $1000 \mu \mathrm{mol} / \mathrm{l}$

(E) Continuous venovenous hamofiltration is contraindicated in patients with deep vein thrombosis

6. Which of the following may cause a rapidly progressive glomerulonephritis?

(A) Systemic lupus erythematosus

(B) ANCA associated vasculitis

(C) Diabetic nephropathy

(D) Cryoglobulinaemia

(E) Rhabdomyolysis 
7. Contrast nephropathy:

(A) Peak creatinine levels usually occur the day after the procedure

(B) ACE-I are renoprotective and should be continued around the time of the procedure

(C) High osmolar contrast medium is least nephrotoxic

(D) Pre-hydration with normal saline may reduce the risk of contrast nephropathy

(E) Diabetic patients are more at risk

\section{Authors' affiliations}

A C Fry, K Farrington, Department of Renal Medicine, Lister Hospital, Stevenage, Hertfordshire, UK

Funding: none.

Competing interests: none declared

\section{REFERENCES}

1 Hou SH, Bushinsky DA, Wish JB, et al. Hospital-acquired renal insufficiency: a prospective study. Am J Med 1983;74:243-8.

2 Feest TG, Round A, Hamad S. Incidence of severe acute renal failure in adults: results of a community based study. BMJ 1993;306:481-3.

3 Thadhani R, Pascual M, Bonventre JV. Acute renal failure. N Engl J Med 1996; 334: 1448-60

4 Klahr S, Miller SB. Acute oliguria. N Engl J Med 1998;338:671-5.

5 Bellomo R, Ronco C, Kellum JA, and the ADQI workgroup, et al. Acute renal failure-definition, outcome measures, animal models, fluid therapy and information technology needs: the Second International Consensus Conference of the Acute Dialysis Quality Initiative (ADQI) Group. Crit Care 2004;8:R204-12.

6 Brezis M, Rosen S. Hypoxia of the renal medulla-its implications for disease. N Engl J Med 1995;332:647-55.

7 Bonventre JV, Weinberg JM. Recent advances in the pathophysiology of ischaemic acute renal failure. J Am Soc Nephrol 2003;14:2199-210.

8 Schrier RW, Wang W, Poole B, et al. Acute renal failure: definitions, diagnosis, pathogenesis, and therapy. J Clin Invest 2004;114:5-14.

9 Rossert JA, Fischer EA. Acute interstitial nephritis. In: Johnson RJ, Feehally J, eds. Comprehensive clinical nephrology. 2nd ed. London: Mosby, 2003:769-7

10 Firth JD. Medical treatment of acute tubular necrosis. Q J Med 1998:91:321-3.

11 Maillet JP, Pelle-Francoz D, Laville M, et al. Nondilated obstructive acute renal failure: diagnostic procedures and therapeutic management. Radiology 1986; 160:659-62

12 Spital A, Valvo JR, Segal AJ. Nondilated obstructive uropathy. Urology 1988;31:478-82.

13 Ahee $\mathbf{P}$, Crowe AV. The management of hyperkalaemia in the emergency department. J Accid Emerg Med 2000;17:188-91.

$14 \mathrm{Kim}$ H-J, Han S-W. Therapeutic approach to hyperkalaemia. Nephron 2002;92(suppl 1):33-40.

15 Throssell D. Hyperkalaemia. In: Glynne P, Allen A, Pusey C, eds. Acute renal failure in practice. London: Imperial College Press, 2002:156-62.

16 Weiner ID, Linas SL, Wingo CS. Disorders of potassium metabolism. In: Johnson RJ, Feehally J, eds. Comprehensive clinical nephrology. 2nd ed. London: Mosby, 2003:109-21

17 Kamel KS, Wei C. Controversial issues in the treatment of hyperkalaemia. Nephrol Dial Transplant 2003;18:2215-18.

18 Kemner MJ, Harps E, Müller-Wiefel DE. Hyperkalaemia: therapeutic options in acute and chronic renal failure. Clin Nephrol 1996;46:67-9

19 Kraut JA, Kurtz I. Use of base in the treatment of severe acidaemic states. Am J Kidney Dis 2001;38:703-27.

20 Finfer S, Bellomo R, Boyce $\mathrm{N}$ et al. A comparison of albumin and saline for fluid resuscitation in the intensive care unit. N Engl J Med 2004;350:2247-56.

21 Cochrane Injuries Group albumin Reviewers. Human albumin administration in critically ill patients: systematic review of randomised controlled trials. BMJ 1998;317:235-40.

22 Bellomo R, Chapman M, Finfer S, et al. Low-dose dopamine in patients with early renal dysfunction: a placebo-controlled randomised trial. Lancet 2000;356:2139-43

23 Debaveye YA, Van de Berghe GH. Is there still a place for dopamine in the modern intensive care unit? Anesth Analg 2004;98:461-8.

24 Galley HF. Renal-dose dopamine: will the message now get through? Lancet 2000;356:2112.

25 Shilliday IR, Quinn KJ, Allison MEM. Loop diuretics in the management of acute renal failure: a prospective double-blind, placebo-controlled, randomised study. Nephrol Dial Transplant 1997;12:2592-6.

26 Mehta RL, Pascual MT, Soroko, S, et al. Diuretics, mortality, and nonrecovery of renal function in acute renal failure. JAMA 2002;288:2547-53

27 Uchino S, Doig GS, Bellomo R, et al. Diuretics and mortality in acute renal failure. Crit Care Med 2004;32:1669-77.
28 Lameire N, Vanholder R, Van Biesen W. Loop diuretics for patients with acute renal failure: helpful or harmful? JAMA 2002;288:2599-601

29 Harris KPG. Urinary tract obstruction. In: Johnson RJ, Feehally J, eds. Comprehensive clinical nephrology. 2nd ed. London: Mosby, 2003:745-58.

30 Lameire N, Van Biesen W, Vanholder R. Acute renal failure. Lancet 2005;365:417-30

31 Walker R. Renal tract obstruction and acute renal failure. In: Glynne P, Allen A, Pusey C, eds. Acute renal failure in practice. London: Imperial College Press, 2002:437-50.

32 Chan LN. Nutritional support in acute renal failure. Curr Opin Clin Nutr Metab Care 2004;7:207-12.

33 Bozfakioglu S. Nutrition in patients with acute renal failure. Nephrol Dial Transplant $2001 ; 16(\mathrm{~S} 6): 21-2$

34 Bellomo R, Ronco C. Indications and criteria for initiating renal replacement therapy in the intensive care unit. Kidney Int 1998;53(suppl 66):106-9.

35 Phu NH, Hien TT, Mai NTH, et al. Haemofiltration and peritoneal dialysis in infection-associated acute renal failure in Vietnam. N Engl J Med 2002; 347:895-902.

36 Forni LG, Hilton PJ. Continuous haemofiltration in the treatment of acute renal failure. N Engl J Med 1997;336:1303-9.

37 Van Biesen W, Vanholder R, Lameire N. Dialysis strategies in critically ill acute renal failure patients. Curr Opin Crit Care 2003;9:491-5.

38 Tonelli M, Manns B, Feller-Kopman D. Acute renal failure in the intensive care unit: a systematic review of the impact of dialytic modality on mortality and renal recovery. Am J Kidney Dis 2002;40:875-85.

39 Schiffl H, Lng SM, Fischer R. Daily haemodialysis and the outcome of acute renal failure. N Engl J Med 2002;346:305-10.

40 Subramanian S, Venkataraman R, Kellum JA. Influence of dialysis membranes on outcomes in acute renal failure: a meta-analysis. Kidney Int 2002;62:1819-23

41 Alonso A, Lau J, Jaber BL. Biocompatible haemodialysis membranes for acute renal failure. Cochrane Library. Issue 2. Chichester: Wiley, 2005.

42 Abdeen O, Mehta RL. Dialysis modalities in the intensive care unit. Crit Care Clin 2002; 18:223-47.

43 Lameire N, Van Biesen W, Vanholder R. Dialysing the patient with acute renal failure in the ICU: the emperor's clothes? Nephrol Dial Transplant $1999 ; 14: 2570-3$

44 Pusey CD, Rees AJ, Evans DJ, et al. Plasma exchange in focal necrotising glomerulonephritis without anti-GBM antibodies. Kidney Int 1991;40:757-63.

45 Tepel M, van der Giet M, Schwarzfeld C, et al. Prevention of radiographiccontrast-agent-induced reductions in renal function by acetylcysteine. N Engl J Med 2000;343:180-4

46 Merten GJ, Burgess WP, Gray LV, et al. Prevention of contrast-induced nephropathy with sodium bicarbonate: a randomised controlled trial. JAMA 2004; 291:2328-34.

47 Aspelin P, Aubry P, Fransson SG, et al. Nephrotoxic effects in high-risk patients undergoing angiography, N Engl J Med 2003:348:491-9.

48 Asif A, Epstein M. Prevention of radiocontrast-induced nephropathy. An J Kidney Dis 2004;44:12-24.

49 Vanholder R, Sever MS, Erek E, et al. Rhabdomyolysis. J Am Soc Nephrol 2000;11:1553-61.

50 Glynne P, Allen A. Rhabdomyolysis. In: Glynne P, Allen A, Pusey C, eds. Acute renal failure in practice. London: Imperial College Press, 2002:296-308.

51 Brown CVR, Rhee P, Chan L, et al. Preventing renal failure in patients with rhabdomyolysis: do bicarbonate and mannitol make a difference? J Trauma 2004:56:1191-6.

52 Ginés P, Guevara M, Arroyo V, et al. Hepatorenal syndrome. Lancet 2003;362:1819-27.

53 Schrier RW, Wang W. Acute renal failure and sepsis. N Engl J Med 2004;351:159-69.

54 Block CA, Manning HL. Prevention of acute renal failure in the critically ill. Am J Respir Crit Care Med 2002;165:320-4

55 Sharples EJ, Patel N, Brown P, et al. Erythropoietin protects the kidney against the injury and dysfunction caused by ischaemia-reperfusion. J Am Soc Nephrol 2004;15:2240-1.

56 Humes HD, Weitzel WF, Bartlett RH, et al. Initial clinical results of the bioartificial kidney containing human cells in ICU patients with acute renal failure. Kidney Int 2004;66:1578-88.

57 Bhandari S, Turney JH. Survivors of acute renal failure who do not recover renal function. QJM 1996:89:415-21.

58 Noble JS, MacKirdy FN, Donaldson SI, et al. Renal and respiratory failure in Scottish ICUs. Anaesthesia 2001;56:124-9.

59 Turney JH, Marshall DH, Brownjohn AM, Ellis CM, Parsons FM. The evolution of acute renal failure 1956-1988. QJM 1990;74:83-104.

60 Levy EM, Viscoli CM, Horwitz RI. The effect of acute renal failure on mortality. JAMA 1996;275:1489-94.

61 Metnitz PGH, Krenn CG, Heinz S, et al. Effect of acute renal failure requiring renal replacement therapy on outcome in critically ill patients. Crit Care Med 2002:30:2051-8.

62 Kaufman J, Dhakal M, Patel B, et al. Community-acquired acute renal failure. Am J Kidney Dis 1991;17:191-8.

63 Turney JH. Acute renal failure-a dangerous condition. JAMA 1996;275:1516-17.

\section{ANSWERS}

1. (A) T, (B) F, (C) T, (D) F, (E) F; 2. (A) F, (B) T, (C) T, (D) F, (E) F; 3. (A) F, (B) F, (C) T, (D), F, (E), T; 4. (A) T, (B) F, (C) F, (D) F, (E) F; 5. (A) F, (B) F, (C) T, (D) F, (E) F; 6. (A) T, (B) T, (C) T, (D) T, (E) F; 7. (A) F, (B) F, (C) F, (D) T, (E) T. 